\title{
Erratum to: Maintenance of drug metabolism and transport functions in human precision-cut liver slices during prolonged incubation for 5 days
}

\author{
Viktoriia Starokozhko ${ }^{1}$ D - Suresh Vatakuti ${ }^{1} \cdot$ Bauke Schievink $^{2}$. \\ Marjolijn T. Merema ${ }^{1} \cdot$ Annika Asplund $^{3} \cdot$ Jane Synnergren $^{3} \cdot$ Anders Aspegren $^{4}$. \\ Geny M. M. Groothuis ${ }^{1}$
}

Published online: 30 November 2016

(C) Springer-Verlag Berlin Heidelberg 2016

\section{Erratum to: Arch Toxicol \\ DOI 10.1007/s00204-016-1865-x}

In the original publication, the name of the third author has been published inadvertently. The authors would like to correct the name to 'Bauke Schievink'.

The original article has been updated accordingly.

The online version of the original article can be found under doi:10.1007/s00204-016-1865-x.

Viktoriia Starokozhko

v.starokozhko@rug.nl

1 Division of Pharmacokinetics Toxicology and Targeting, Groningen Research Institute for Pharmacy, University of Groningen, Groningen, The Netherlands

2 Department of Clinical Pharmacy and Pharmacology, University of Groningen, University Medical Center Groningen, Groningen, The Netherlands

3 School of Biosciences, Systems Biology Research Center, University of Skövde, 54128 Skövde, Sweden

4 Takara Bio Europe AB, Gothenburg, Sweden 